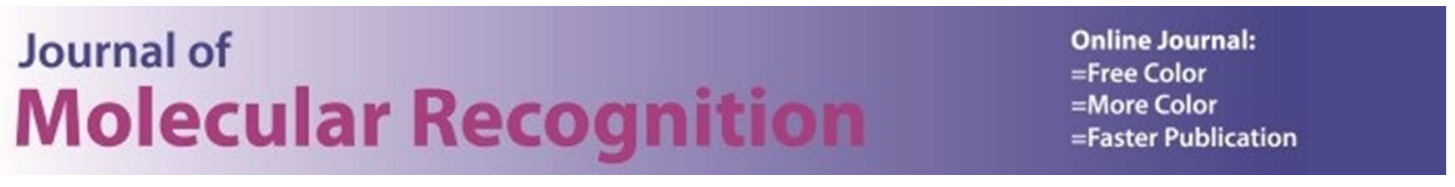

\title{
Multispecificity of a recombinant anti-ras monoclonal antibody
}

\begin{tabular}{|r|l|}
\hline Journal: & Journal of Molecular Recognition \\
\hline Manuscript ID & JMR-17-0049.R1 \\
\hline Wiley - Manuscript type: & Research Article \\
\hline Complete List of Authors: & $\begin{array}{l}\text { Schrader, John; University of British Columbia, The Biomedical Research } \\
\text { Centre } \\
\text { McLean, Gary; London Metropolitan University, Cellular and Molecular } \\
\text { Immunology Research Centre }\end{array}$ \\
\hline Keywords: & Recombinant monoclonal antibody, specificity, polyreactivity, ras \\
\hline \multicolumn{2}{|r}{} \\
\hline
\end{tabular}


Multispecificity of a recombinant anti-ras monoclonal antibody

John W Schrader ${ }^{1}$ and Gary R McLean ${ }^{2,3}$ *

${ }^{1}$ The Biomedical Research Centre, University of British Columbia, Vancouver BC Canada

${ }^{2}$ Cellular and Molecular Immunology Research Centre, London Metropolitan University, London, UK

${ }^{3}$ National Heart and Lung Institute, Imperial College London, London UK

*Corresponding author: CMIRC, London Metropolitan University, 166-220 Holloway Road, London UK N7 8DB (g.mclean@londonmet.ac.uk)

\begin{abstract}
Recombinant monoclonal antibodies (Ab's) have widespread application as research tools, diagnostic reagents and as biotherapeutics. Whilst studying the cellular molecular switch protein $\mathrm{m}$-ras, a recombinant monoclonal antibody to $\mathrm{m}$-ras was generated for use as a research tool. Antibody genes from a single rabbit B cell secreting IgG to an m-ras specific peptide sequence were expressed in mammalian cells and monoclonal rabbit IgG binding was characterized by ELISA and peptide array blotting. Although the monoclonal $\mathrm{Ab}$ was selected for specificity to m-ras peptide it also bound to both recombinant full-length $\mathrm{m}$-ras and h-ras proteins. The cross reactive binding of the monoclonal $\mathrm{Ab}$ to $\mathrm{h}$-ras was defined by peptide array blot revealing that the $\mathrm{Ab}$ showed preference for peptide sequences containing multiple positively charged amino acid residues. These data reinforce the concept of antibody multispecificity through multiple interactions of the $\mathrm{Ab}$ paratope with diverse polypeptides. They also emphasize the importance of immunogen and $\mathrm{Ab}$ selection processes when generating recombinant monoclonal Ab's.
\end{abstract}

Keywords: Recombinant monoclonal antibody; specificity; polyreactivity; ras

\title{
Introduction
}

Antibodies (Ab's) are glycoproteins of the immunoglobulin superfamily that are produced by B lymphocytes to foreign antigens (Ag's) (for review see [1]). During immune responses Ab's are produced in a polyclonal fashion to Ag's by numerous B cell although individual B cells produce a single Ab clonotype. Polyclonal Ab's therefore refer to the mixture of Ab's found in biological fluids, such as serum, that are produced by many B cells. Thus, polyclonal Ab's are defined as having a large number of specificities but tend to be enriched for a particular Ag, often following immunization or exposure to the Ag [2]. Importantly, in 1975 the advent of hybridoma technology permitted the isolation of B cell clones allowing for continued production of monoclonal Ab's [3]. Thus, monoclonal Ab's are a single clone of Ab's thought as being superior to polyclonal Ab's due to their selectivity and specificity for single Ag's. Over the last forty years, exploitation of their 
ability to specifically recognise, immobilise, destroy or clear such Ag's has seen both polyclonal and monoclonal Ab's transformed into diagnostic markers, laboratory tools and powerful therapeutics and prophylactics for human diseases [4-6]. These Ab's are usually of the IgG isotype due to their generalised function in vitro and relatively long half-life and superior effector function in vivo as opposed to that of the $\operatorname{IgA}$ and $\operatorname{IgM}$ isotypes which have more specialised functions.

Ab's have enjoyed great utility in numerous applications within research laboratories, diagnostic laboratories and in clinical settings [7]. Laboratory methods such as immunohistochemistry, immunofluorescence, western blot, immunoprecipitation, enzyme-linked immunoassay and flow cytometry that rely exclusively on Ab's are routine in most biomedical laboratories. In clinical settings, Ab's are useful diagnostic markers providing evidence of infectious disease status and immunisation efficacy. Additionally, polyclonal Ab preparations have long been used as therapeutic/prophylactic agents with immune globulin (IVIg) still in frequent use [8]. These applications of polyclonal Ab's rely on their specificity and sensitivity but it is becoming apparent that Ab's are not as entirely specific or selective as first envisioned. The hope was that much of the difficulty associated with the mixtures of $\mathrm{Ab}$ specificity found with polyclonal reactivity could be alleviated through the use of monoclonal Ab's [7]. Thus, many monoclonal Ab's are now available for laboratory assays, having replaced polyclonal Ab's and have become the fastest growing area in biotherapeutics with approximately 40 now approved by the United States' FDA and Europe's EMA [9].

Since the original description of mouse monoclonal Ab's in 1975 [3] the various methods for monoclonal $\mathrm{Ab}$ generation has amplified and the generation of numerous species of Ab's including human clones is now possible [9]. Recombinant Ab's, where the genes encoding a monoclonal Ab are expressed in vitro, are now becoming a dominant form of Ab production. It is relatively simple to produce recombinant Ab's in a variety of expression systems such as mammalian, bacterial, yeast, insect, or plant cells once the original B cell clone has been selected [10], although glycosylation is known to vary and this may affect $\mathrm{Ab}$ functions [11]. Nevertheless, the use of 
recombinant monoclonal Ab's and traditional monoclonal Ab's has improved some of the unwanted reactivity profiles that have been associated with polyclonal Ab's, however monoclonal Ab's are not exempt from cross reactivity and some have the ability to bind to a variety of unrelated self or foreign antigens through what is known as polyreactivity [12]. In fact, the term "Ab cross reactivity" suggests that each Ab binds to a primary epitope and that binding to any other epitope is an unwanted example of a lack of specificity. As summarized recently by Van Regenmortel [13], a variety of mechanisms can explain how a polyspecific Ab can bind to multiple epitopes. Therefore, an $\mathrm{Ab}$ combining site, or paratope, may be a rigid structure but allow interaction with different epitopes at different subsites in a process called rigid adaptation [14]. Alternatively, Ab paratopes may adopt multiple conformations through flexibility and bind to many different epitopes via an induced fit mechanism [15]. Finally, according to the differential ligand positioning model, a flexible $\mathrm{Ab}$ structure can bind diverse epitopes at different regions of the Ab combining site [16]. Together, these mechanisms can help explain the observed polyspecificity of numerous monoclonal Ab's and the fact that polyclonal antiserum can sometimes outperform certain monoclonal Ab's in specificity rankings.

In the current study, a recombinant monoclonal $\mathrm{Ab}$ to m-ras (anti-m-ras) was generated by immunization of a rabbit with an m-ras C-terminal peptide followed by isolation of a single rabbit peripheral blood B cell secreting specific IgG and recombinant expression of the rabbit Ab genes in mammalian cells. We were using this monoclonal Ab to study ras proteins, an important family of intracellular molecular switches with roles in the regulation of cell growth, survival and differentiation [17]. The most well-known of these are the p21 ras proteins h-ras, n-ras, and k-ras that are often mutated in human cancers and are highly homologous to ras family members r-ras, mras and rap and ral proteins [17]. Here we show that although the immunogen sequence used for immunization was specific to m-ras and the rabbit antisera and the recombinant anti-m-ras IgG bound to this peptide sequence somewhat specifically, upon further investigation it was found that the anti-m-ras IgG displayed cross reactivity. Thus, it bound both recombinant $\mathrm{m}$-ras and the related 
recombinant h-ras equally well despite the latter protein not containing the immunogen sequence. Further investigations reported within have revealed the potential molecular basis for the polyspecificity of this recombinant monoclonal $\mathrm{Ab}$. These data add to the growing body of identified monoclonal $\mathrm{Ab}$ polyspecificity and consequently have important implications for interpretation of results obtained when relying on the specificity of monoclonal Ab's.

\section{Materials and Methods}

Peptides and recombinant proteins

Polypeptides used for immunization and used as antigens in ELISA and array blot experiments were generated by solid phase synthesis and were a gift from Dr Ian Clarke-Lewis (Biomedical Research Centre, Vancouver BC, Canada). Recombinant human m-ras and h-ras used as antigens in ELISA experiments were produced in bacteria as GST fusion proteins and were a gift from Gary Wilson (Biomedical Research Centre, Vancouver BC, Canada). The GST sequence was added to the N-terminus of m-ras and h-ras through the use of pGEX expression systems and was used as an affinity tag to purify the recombinant proteins using immobilized reduced glutathione and was not cleaved from the purified product [18].

\section{Immunisation of rabbits and antisera}

Rabbits were injected with peptides corresponding to residues 187-204 (KKKTKWRGDRATGTHKLQ) of m-ras fused to the C-terminus of the tetanus toxoid epitope QYIKANSKFIGITEL as described previously [19]. Antisera was obtained from peripheral blood and affinity purified as described [19].

\section{Rabbit monoclonal Ab generation}


The selected lymphocyte antibody method (SLAM) [20] was performed using peripheral blood lymphocytes from an immunized rabbit to isolate single rabbit B cells secreting IgG specific for mras. Antibody variable (V) region gene segments were cloned from a single cell as described previously [20] and expressed as rabbit IgG following insertion into mammalian immunoglobulin expression vectors [21] modified to contain rabbit IgG constant (C) region genes [22]. Rabbit IgG was isolated from culture supernatants of transiently transfected HEK293 cells as described [22]. The anti-m-ras V region cDNA sequences were obtained by Sanger sequencing of the Ig expression plasmids using a CMV promoter primer at the University of British Columbia NAPS unit (Vancouver, BC, Canada).

\section{Enzyme-linked immuosorbent assay (ELISA)}

ELISA was performed as described previously [22]. Briefly, flat bottomed 96-well microtiter plates (Nunc Maxisorp) were coated with peptides or recombinant protein at a concentration of 1 $\mu \mathrm{g} / \mathrm{ml}$ in phosphate buffered saline (PBS) overnight at $4^{\circ} \mathrm{C}$. The coating of different sized antigens at the same concentration will result in different densities of antigen depending upon their molecular weight and in-turn alters the binding curves obtained. Plates were then blocked with PBS containing $2 \%$ bovine serum albumin (BSA) at room temperature for 2 hours before addition of dilutions of rabbit antisera, culture supernatants containing rabbit IgG or affinity purified rabbit IgG. After an overnight incubation at $4{ }^{\circ} \mathrm{C}$ the plates were washed with PBS containing $0.5 \%$ tween 20 (PBST) and the bound rabbit IgG detected with goat anti-rabbit IgG alkaline phosphatase (AP) conjugate for 1 hour at $37^{\circ} \mathrm{C}$. Plates were washed again with PBST and developed using paranitrophenyl phosphate (pNPP) substrate with the optical density measured at $405 \mathrm{~nm}$ in a spectrophotometer.

\section{Peptide spot array blot}

An overlapping peptide array corresponding to the primary amino acid sequences of human mras and h-ras was prepared by spotting peptides of 10 amino acid residues onto nitrocellulose 
membranes (a gift from Dr Peter Schubert, Biomedical Research Centre, Vancouver, BC, Canada). Each subsequent spot overlapped with the sequence of the previous peptide spot by 8 amino acids such that the second peptide spot began with amino acid 3 of the ras sequences and the third peptide spot began with amino acid 5 etc. Thus the h-ras polypeptide was covered by 91 unique peptide spots whereas m-ras required 99 spots for complete coverage. Membranes were blocked with PBS containing 5\% skim milk powder before the addition of diluted rabbit anti-m-ras $\mathrm{Ab}$ and incubated at $4{ }^{\circ} \mathrm{C}$ overnight with shaking. The membranes were washed with PBST before the addition of secondary $\mathrm{Ab}$ anti-rabbit IgG horseradish peroxidase (HRP) conjugate. Washed membranes were covered with ECL substrate for 1 minute and spots developed by exposure to X-ray film.

\section{Results}

C-terminal regions of human m-ras and h-ras protein primary sequences were inspected for selection of peptides that displayed regions that could be exploited to generate m-ras specific Ab's. Despite overall identity of $48.6 \%$ between human m-ras and h-ras, the C-terminal regions display much weaker identity (Figure 1A). Therefore, the region corresponding to amino acids 187-204 of human m-ras displayed just $21.7 \%$ identity to h-ras and was selected as being unique to m-ras (Figure 1B). Performing a NCBI Blast search using the 187-204 sequence of m-ras detected only mras protein sequences from a variety of species, including rabbit, but not to other related ras proteins. It was therefore hypothesized that this sequence might be an epitope capable of eliciting m-ras specific Ab's as the use of C-terminal peptides in immunization strategies results in Ab's that bind native protein [23].

Antisera was obtained from a rabbit immunized with the synthesized peptide corresponding to residues 187-204 of m-ras which had been fused to the C-terminus of the tetanus toxoid epitope to promote immunogenicity in rabbits. ELISA was performed to determine binding specificity and titer of this antisera. As shown in Figure 2, the rabbit antisera was very specific for the m-ras 
peptide used as the immunogen with IgG binding still detectable at a dilution of approximately 1:500 whereas IgG binding to the corresponding h-ras peptide was very weak at this dilution and was only observed at dilutions of antisera less than 1:10.

Peripheral blood B cells were then obtained from the rabbit to generate an m-ras specific monoclonal Ab by the selected lymphocyte antibody method (SLAM) [20]. Sheep red blood cells coated with the m-ras peptide were used to identify and isolate single rabbit B cells producing specific antibodies. The immunoglobulin V regions of one selected clone were amplified by PCR and cloned into immunoglobulin expression vectors [21] that contained rabbit $\mathrm{C}$ regions [22] to generate a rabbit IgG clone specific for m-ras. The antibody $\mathrm{V}$ region predicted amino acid sequences obtained from plasmid cDNA sequencing were aligned with the nearest rabbit germ-line $\mathrm{V}$ region segments as defined by IMGT/V-QUEST [24]. These amino acid sequences and junctional rearrangements are shown in Figure 3. Analysis of the sequences revealed that the m-ras Vk sequence (Genbank accession number KX858713) was composed of the rearranged rabbit germline IGKV1S2*01 and IGKJ1-2*01 alleles with numerous somatic mutations clustered in the complementarity determining regions (CDR's) which also corresponded to predicted antigenbinding regions (ABRs) as determined by Paratome analysis $[25,26]$ (Fig 3A). The m-ras Vh sequence (Genbank accession number KX858712) was composed of the rearranged rabbit germline IGHV1S69*01, IGHD8-1*01 and IGHJ4*01 alleles with evidence of CDR-focused somatic mutations. The Vh CDR3 in particular contained at least five somatic mutations that resulted in negatively charged aspartic acid residues (Asp, D) within the sequence. Further analyses of the Vh and $\mathrm{Vk}$ junctions indicated that there was also the likelihood of significant junctional insertions in CDR3 of both the m-ras Vh and the m-ras Vk regions (Fig 3B).

The monoclonal rabbit anti-m-ras IgG was produced by transient transfection of HEK293 cells with plasmids $\mathrm{pLC}-\mathrm{rbC} \kappa(\mathrm{m}-\mathrm{ras})$ and $\mathrm{pHC}-\mathrm{rbC} \gamma 1(\mathrm{~m}-\mathrm{ras})$ that contain the genes for rabbit anti-m-ras kappa light chains and gammal heavy chains respectively. Rabbit IgG in culture supernatants was then tested for binding specificity by ELISA. Binding to several peptides corresponding to C- 
terminal regions of m-ras (KKKTKWRGDRATGTHKLQ), $\quad$ r-ras2 (ECPPSPEPTRKEKDKKGCHC) and h-ras (KLRKLNPPDESGPGCM) was first assessed. Significant and specific binding of anti-m-ras IgG was observed to the m-ras peptide but not to peptides of r-ras2 and h-ras (Fig 4). Control culture supernatant of untransfected HEK293 cells showed no binding to the m-ras peptide. Having confirmed that the anti-m-ras monoclonal IgG was specific for the m-ras C-terminal peptide, its ability to bind to recombinant ras proteins was next determined. Interestingly, we found that anti-m-ras rabbit IgG binds equally well to ELISA plates coated with m-ras or h-ras recombinant proteins (Fig 5A), despite the latter protein not containing the immunogen epitope sequence. In other sandwich ELISA experiments to confirm these data, these ras recombinant proteins were first captured with the plate-bound anti-ras scFv Y13-259 [27] and then detected using the rabbit anti-m-ras monoclonal $\mathrm{Ab}$. It was found again that both m-ras and h-ras were bound equally by the anti-m-ras monoclonal $\mathrm{Ab}$ (Fig 5B) even though a significantly weaker signal was detected to both proteins, possibly due to steric hindrance by the Y13-259 capture $\mathrm{Ab}$.

Concerned about the specificity of the anti-m-ras monoclonal $\mathrm{Ab}$, the potential epitopes of both m-ras and h-ras that bound the rabbit IgG were mapped using an overlapping peptide array corresponding to the primary amino acid sequences of human m-ras and h-ras. Supplementary Figure 1 displays the peptides sequences spanning the m-ras and h-ras polypeptides. The data obtained with the ras peptides array and the anti-m-ras monoclonal $\operatorname{IgG}$ is shown in Figure 6. It can be readily seen that binding of the anti-m-ras IgG to peptide sequences of both h-ras and m-ras, above background reactivity with secondary $\mathrm{Ab}$ alone was observed. Additionally, there was clearly more binding to m-ras sequences than was detected to h-ras sequences. Thus, the anti-m-ras IgG bound strongly to the m-ras peptides spanning the C-terminal region (peptides 86-98) as expected but also to peptides spanning almost the entire remainder of the primary sequence (peptides 5-29, $53-73,77-82$ ) with a few notable exclusions (peptides 1-3, 6, 14, 19, 20, 23-27, 58, 59, 72, 80, 87 were not bound well). The region covered by peptides $30-52$ was not bound by the anti-m-ras IgG 
except for some weak binding to peptides $34,42,46,48$ and 51. Conversely, the anti-m-ras IgG bound to less of the h-ras primary sequence, favoring smaller regions covered by peptides proximal to the N-terminus (peptides 2-7 and 17-22), the middle of the sequence (peptides 40-57) and the Cterminal region (peptides 71-85). There were large areas of the sequence not bound at all with notable exclusions being covered by peptides $24-39$, 58-70 and the C-terminus $86-91$. The strongest binding was observed to peptides $21,22,40,49-51,57,72$ and 81-84. To confirm these data visually by relating to the ras primary sequence, an analysis was performed comparing spot intensity to peptide sequence within the m-ras and h-ras sequence. Figure 7 shows these data as bar graphs representing spot intensity as a function of peptide sequence. Here it is readily observed that sections of the m-ras sequence covered by peptides 5-29 and 53-98 contain numerous peptides that are bound strongly whereas binding is restricted to much smaller regions $(21-22,49-51,81-84)$ of h-ras with several notable single peptides $(40,57,72)$ also bound well. The common feature of the majority of the h-ras and m-ras peptides bound by anti-m-ras IgG was the presence of positively charged amino acid residues arginine, lysine, and histidine. For example, binding to h-ras peptides was strongest in the regions of peptides 49-51, a sequence containing two lysine residues and two arginine residues, and also to peptides 81-84 that are particularly rich in both lysine and arginine residues. For m-ras, the strongest binding was observed in regions containing peptides 53-73 (13 positively charged residues) and 86-98 (the C-terminal lysine-rich region). However, a minority of m-ras and h-ras peptides are not bound well even though they do contain at least one positively charged residue. For example h-ras peptides 19, 20, 58, 59, 73 and 74 each contain two positively charged residues but are not bound. This feature is less evident with m-ras, however peptides 83-85 are not bound despite containing positive charged residues. In addition, some peptides are bound strongly although they do not contain positively charged residues (e.g. h-ras peptide 22; m-ras peptides 21, 28 and 29). These data offer potential explanations in molecular terms as to how the anti-m-ras monoclonal $\mathrm{Ab}$ was able to bind to both $\mathrm{m}$-ras and h-ras recombinant proteins. 


\section{Discussion}

Here we have investigated the polyspecificity of a recombinant monoclonal $\mathrm{Ab}$ originally selected for binding to an m-ras specific peptide sequence that was rich in positively charged amino acid residues. The initial aim was to identify an m-ras specific polypeptide sequence and to use this to raise an m-ras specific monoclonal Ab. However, we found that the anti-m-ras monoclonal IgG bound to both h-ras and m-ras polypeptides despite being selected for binding to an m-ras specific C-terminal peptide sequence and the rabbit antisera that this clone was derived from was very specific for the m-ras peptide. More detailed epitope analyses demonstrated that the anti-m-ras monoclonal IgG also bound to numerous linear peptide sequences within both the m-ras and h-ras primary sequences and that the majority of these peptides contained positively charged amino acid residues, partially explaining the Ab polyspecificity. This ability of a monoclonal Ab to bind numerous and diverse peptide sequences that we describe here highlight the role of the $\mathrm{Ab}$ combining site in providing a structural paratope that has the ability to recognize and bind many different epitopes, effectively expanding the $\mathrm{Ab}$ repertoire to allow recognition of any antigen. The results also demonstrate that the precise analysis of data obtained when using monoclonal Ab's for laboratory investigations involving protein detection is necessary as polyspecificity could give rise to numerous interpretations.

A common approach for generating antisera or monoclonal Ab's to a newly discovered protein, or to one where no suitable $\mathrm{Ab}$ reagents exist, is to immunize animals with a unique synthetic peptide found at the C-terminus of the primary sequence. This approach often results in specific Ab's that bind the native protein [23] and that have bioactivity in vivo [28] although cross reactivity or polyspecificity is rarely investigated. In this study we identified a C-terminal region of human mras that was unique amongst the known ras protein sequences and it was hypothesized that this sequence would elicit m-ras specific Ab's when used as an immunogen. In fact, we found that antisera obtained from animals immunized with the m-ras peptide bound the m-ras immunogen peptide much more strongly than it did to the corresponding h-ras peptide sequence that is only $21 \%$ 
identical in primary sequence. Antisera binding to the h-ras peptide was detectable when undiluted but was lost at dilutions greater than 1:4 whereas binding to the m-ras peptide remained strong, even at antisera dilutions of 1:500. Thus, it was concluded that the antisera was "specific for m-ras" and would be a useful starting point for monoclonal $\mathrm{Ab}$ development. Following selection of a monoclonal $\mathrm{Ab}$ from this pool of Ab's we found the $\mathrm{Ab}$ was capable of binding equally well to both the m-ras and h-ras polypeptides. Interestingly, the anti-m-ras monoclonal $\mathrm{Ab}$ did not display binding to the homologous lysine-rich peptide sequences of h-ras and r-ras2 whilst binding strongly to the m-ras immunizing peptide as shown in Figure 4. These important results are suggestive of these peptides not being promiscuously sticky, at least in ELISA experiments, and that the Ab is also not a universal binder. Taken together, these findings reinforce the often observed phenomenon that with protein Ag's, polyclonal Ab's and antisera are almost always more specific than monoclonal Ab's. This is because protein Ag's contain multiple epitopes and that all Ab's to some degree are polyspecific, having the ability to bind to several and sometimes quite diverse epitopes. Thus, a polyclonal mixture of Ab's will bind to numerous epitopes polyspecifically but the collective cross reactivity will be diluted out in such mixtures of Ab's due to the common specificity to an immunodominant epitope. This phenomenon does not readily occur with single monoclonal Ab's due to their selectivity for shorter epitopes and possible polyspecificity, theoretically explaining why polyclonal $\mathrm{Ab}$ mixtures often tend to be more specific than individual monoclonal Ab's [13]. Our data shows that, even though the polyclonal antisera can bind both the m-ras and h-ras peptides, it is much more specific for the m-ras peptide whereas the isolated monoclonal $\mathrm{Ab}$ from that mixture is far less selective, binding to numerous and diverse peptide sequences.

It is well established that the molecular interactions between $\mathrm{Ab}$ combining sites, which consists of six complementarity determining regions within the $\mathrm{Ab} \mathrm{Vh}$ and $\mathrm{Vl}$ regions, and $\mathrm{Ag}$ epitopes are a series of weak noncovalent chemical bonds such as electrostatic interactions, hydrogen bonds, van der Waals forces, and hydrophobic interactions [29]. In addition, structural 
considerations of both the $\mathrm{Ab}$ paratope and $\mathrm{Ag}$ epitope influence the strength and specificity of these interactions and can contribute to $\mathrm{Ab}$ cross reactivity and polyspecificity [30]. The data we have obtained for this monoclonal $\mathrm{Ab}$ suggest that electrostatic interactions between positively charged epitopes and a negatively charged Ab paratope may be an important factor in influencing the majority of polyspecific interactions and therefore the cross reactivity, although other interactions are also possible as not all peptides appeared to be bound via such electrostatic factors. Thus, immunization with a peptide containing numerous ( 7 out of $18,39 \%$ ) positively charged amino acid residues resulted in selection of an $\mathrm{Ab}$ clone that preferred interactions with such residues. Although it was not anticipated that a preferential selection of Ab's that interact predominantly via these positive charges might occur, in hindsight it does seem likely, and could perhaps be exploited in the future to induce $\mathrm{Ab}$ polyspecificity via directed immunizations if desired.

Analysis of the anti-m-ras $\mathrm{Ab} V$ region CDR sequences revealed that 4 of the $6 \mathrm{CDR}$ 's (LCDR1, LCDR3, HCDR1, HCDR3) contained negatively charged amino acid residues that might interact with positively charged residues in the m-ras and h-ras epitopes. When looking at $\mathrm{Ab}$ paratopes, it is known that the HCDR3 contains the most diversity and forms the majority of contacts of $\mathrm{Ab}$ with $\mathrm{Ag}$ epitopes [31]. Interestingly the HCDR3 of the m-ras $\mathrm{Ab}$ contained numerous ( 5 out of $12,42 \%$ ) negatively charged amino acid residues that most likely resulted from somatic hypermutations brought about by positive selection through Ag binding. Furthermore, no positively charged amino acids were found in any of the 6 CDR's of the anti-m-ras Ab suggesting that these residues were selected against. Clearly, in the absence of structural data confirming the precise interactions between the $\mathrm{Ab}$ paratope $\mathrm{CDR}$ residues and the various epitopes it is pure conjecture that such electrostatic interactions dictate the $\mathrm{Ab}$ polyspecificity, however similar supporting observations have been shown previously with autoreactive monoclonal Ab's in mice [32]. Thus, our analyses of $\mathrm{Ab} \mathrm{V}$ region sequences, coupled with the molecular observations of polyreactivity do help explain the molecular basis of the multispecificity of Ab's in general and 
specifically in this case, the ability of the anti-m-ras monoclonal Ab to bind numerous peptides of both m-ras and h-ras.

When our investigations were expanded to a larger number of peptides we found that the antim-ras IgG could bind to numerous unrelated sequences to the immunizing peptide sequence in addition to similar peptide sequences. The peptide sequences we investigated were based on the primary sequences of both m-ras and h-ras in an effort to understand how the monoclonal $\mathrm{Ab}$ was able to bind both of these proteins equally. As such, we did not investigate binding to unrelated peptide sequences although it can be argued that the homology of m-ras and h-ras is disparate enough to have provided enough diversity in the peptide sequences we analyzed and might reflect cross reactive binding to unrelated polypeptides. The most common feature of the epitopes bound, as outlined previously, was the cationic property of the peptides although other unrelated peptide sequences were also bound. Thus, a minority of peptides appeared to interact with the $\mathrm{Ab}$ in a manner other than through positive/negative charges, perhaps via primary sequence recognition, hydrophobicity, or even a structural basis. These data emphasize the likelihood that this particular monoclonal $\mathrm{Ab}$ is polyspecific with numerous detectable interactions occurring depending on the nature of the epitope. Thus, the anti-m-ras monoclonal $\mathrm{IgG}$ is capable of binding to numerous divergent peptide sequences but that the majority of these contain positively charged amino acid residues $(K, R$, and $H)$, which perhaps not surprisingly, were also enriched in the m-ras immunizing peptide. These data also help explain in molecular terms how the monoclonal $\mathrm{Ab}$ was found to bind to both m-ras and h-ras recombinant proteins.

In this study we performed heterogeneous immunoassays where binding of Ab's in solution to the immobilized Ag/peptide were favored because a consistent approach in analyzing Ab's binding to multiple epitopes was required. These assays therefore serendipitously allowed the description of some molecular details of the monoclonal Ab polyspecificity. Our analysis of the reactivity profile was limited to binding to a related ras recombinant protein and linear peptide sequences contained within ras proteins, although there is significant sequence divergence that would allow for 
extrapolation of these results. It can be argued that our data obtained using small peptides somewhat selects for binding dominated by charged interactions and cannot be more broadly interpreted [33]. However, our initial multispecific observations were observed with larger recombinant ras protein sequences and therefore may be interpreted in a more biological context. It is also possible that the observed polyreactivity may be altered under different binding conditions and Ag availability that are used in other methods. Nevertheless, these differences in Ab behavior based on the analytical method used and the physical nature of the epitope(s) highlights some of the interpretation issues that are associated with the use of monoclonal Ab's in immunoassays. Monoclonal Ab's have found remarkable utility as laboratory reagents, analytical tools and more recently as therapeutic reagents that is largely based on their target selectivity and the specificity of their binding characteristics. It has also been estimated that around half of all commercially obtained Ab's used in laboratories may bind to targets in addition to the one designed for [34]. This may be due to polyspecificity but the issue is often not investigated deeply unless discovered by chance. Additionally, differences in $\mathrm{Ab}$ reactivity profiles exist when using different analytical techniques and applications. Clearly, some Ab's prefer denatured targets to native configurations and others rely on fixing or chemical modifications for optimal specificity and perhaps even polyspecificty.

In summary, our data have described the multispecificity of a recombinant monoclonal $\mathrm{Ab}$ that was discovered by chance. In this case, the data are suggestive of Ab multispecificty being regulated to a large degree by electrostatic interactions between a negatively charged $\mathrm{Ab}$ paratope and positively charged epitopes. Future experiments beyond what is possible in the current manuscript that would prove this theory would involve mutagenesis studies of the acidic residues found in the anti-m-ras $\mathrm{Ab}$ to probe the polyspecificity profile and modifications of basic residues in specific h-ras peptides may to confirm that the molecular nature of the cross reactivity is predominantly due to positive-negative charge interactions. Although this is just one example of $\mathrm{Ab}$ multispecificity, the phenomenon can be generally applied to monoclonal Ab's and question current 
vaccine engineering attempts relying on structure-guided epitope recognition by neutralizing Ab's $[35]$.

\begin{abstract}
Acknowledgements: The authors wish to acknowledge Phil Owen for peptide synthesis, Annette Ehrhardt for rabbit antisera, Gary Wilson for recombinant ras proteins, Peter Schubert for peptide spot array materials, P Rathanaswami for the anti-m-ras clone cDNA and all members of the Schrader laboratory of the Biomedical Research Centre (Vancouver, BC, Canada) for helpful discussions.
\end{abstract}

Author Contributions: GRM conceived and performed the experiments, and wrote the manuscript. JWS assisted with experimental design and edited the manuscript.

Conflicts of Interests: The authors declare they have no conflicts of interests.

\title{
References
}

1. Schroeder, H.W., Jr. and L. Cavacini, Structure and function of immunoglobulins. J Allergy Clin Immunol, 2010. 125(2 Suppl 2): p. S41-52.

2. Cooper, H.M. and Y. Paterson, Production of polyclonal antisera. Curr Protoc Immunol, 2001. Chapter 2: p. Unit 2.4.

3. Kohler, G. and C. Milstein, Continuous cultures of fused cells secreting antibody of predefined specificity. Nature, 1975. 256(5517): p. 495-7.

4. Nelson, P.N., et al., Monoclonal antibodies. Mol Pathol, 2000. 53(3): p. 111-7.

5. Laffly, E. and R. Sodoyer, Monoclonal and recombinant antibodies, 30 years after. Hum Antibodies, 2005. 14(1-2): p. 33-55.

6. Salemi, S., et al., The expanding role of therapeutic antibodies. Int Rev Immunol, 2015. 34(3): p. 202-64.

7. $\quad$ Lipman, N.S., et al., Monoclonal versus polyclonal antibodies: distinguishing characteristics, applications, and information resources. Ilar j, 2005. 46(3): p. 258-68.

8. Hartung, H.P., et al., Clinical applications of intravenous immunoglobulins (IVIg)--beyond immunodeficiencies and neurology. Clin Exp Immunol, 2009. 158 Suppl 1: p. 23-33.

9. Liu, J.K., The history of monoclonal antibody development - Progress, remaining challenges and future innovations. Ann Med Surg (Lond), 2014. 3(4): p. 113-6.

10. Frenzel, A., M. Hust, and T. Schirrmann, Expression of recombinant antibodies. Front Immunol, 2013. 4: p. 217.

11. Batra, J. and A.S. Rathore, Glycosylation of Monoclonal Antibody Products: Current Status and Future Prospects. Biotechnol Prog, 2016.

12. Notkins, A.L., Polyreactivity of antibody molecules. Trends Immunol, 2004. 25(4): p. 174-9. 
13. Van Regenmortel, M.H., Specificity, polyspecificity, and heterospecificity of antibodyantigen recognition. J Mol Recognit, 2014. 27(11): p. 627-39.

14. McFarland, B.J. and R.K. Strong, Thermodynamic analysis of degenerate recognition by the NKG2D immunoreceptor: not induced fit but rigid adaptation. Immunity, 2003. 19(6): p. 803-12.

15. James, L.C., P. Roversi, and D.S. Tawfik, Antibody multispecificity mediated by conformational diversity. Science, 2003. 299(5611): p. 1362-7.

16. Sethi, D.K., et al., Differential epitope positioning within the germline antibody paratope enhances promiscuity in the primary immune response. Immunity, 2006. 24(4): p. 429-38.

17. Ehrhardt, A., et al., Ras and relatives--job sharing and networking keep an old family together. Exp Hematol, 2002. 30(10): p. 1089-106.

18. Harper, S. and D.W. Speicher, Purification of proteins fused to glutathione S-transferase. Methods Mol Biol, 2011. 681: p. 259-80.

19. Ehrhardt, G.R., et al., M-Ras, a widely expressed 29-kD homologue of 21 Ras: expression of a constitutively active mutant results in factor-independent growth of an interleukin-3dependent cell line. Blood, 1999. 94(7): p. 2433-44.

20. Babcook, J.S., et al., A novel strategy for generating monoclonal antibodies from single, isolated lymphocytes producing antibodies of defined specificities. Proc Natl Acad Sci U S A, 1996. 93(15): p. 7843-8.

21. McLean, G.R., et al., Human and murine immunoglobulin expression vector cassettes. Mol Immunol, 2000. 37(14): p. 837-45.

22. McLean, G.R., et al., RIVETS: the recombinant immunoglobulin and viral epitope tag system. J Immunol Methods, 2006. 315(1-2): p. 208-13.

23. Lei, S., M.A. Raftery, and B.M. Conti-Tronconi, Monoclonal antibodies against synthetic sequences of the nicotinic receptor cross-react fully with the native receptor and reveal the transmembrane disposition of their epitopes. Biochemistry, 1993. 32(1): p. 91-100.

24. Brochet, X., M.P. Lefranc, and V. Giudicelli, IMGT/V-QUEST: the highly customized and integrated system for $I G$ and TR standardized $V$-J and $V$-D-J sequence analysis. Nucleic Acids Res, 2008. 36(Web Server issue): p. W503-8.

25. Kunik, V., B. Peters, and Y. Ofran, Structural consensus among antibodies defines the antigen binding site. PLoS Comput Biol, 2012. 8(2): p. e1002388.

26. Kunik, V., S. Ashkenazi, and Y. Ofran, Paratome: an online tool for systematic identification of antigen-binding regions in antibodies based on sequence or structure. Nucleic Acids Res, 2012. 40(Web Server issue): p. W521-4.

27. Lacal, J.C. and S.A. Aaronson, Monoclonal antibody Y13-259 recognizes an epitope of the p21 ras molecule not directly involved in the GTP-binding activity of the protein. Mol Cell Biol, 1986. 6(4): p. 1002-9.

28. Ziltener, H.J., et al., Monoclonal antipeptide antibodies recognize IL-3 and neutralize its bioactivity in vivo. J Immunol, 1988. 140(4): p. 1182-7.

29. Davies, D.R., E.A. Padlan, and S. Sheriff, Antibody-antigen complexes. Annu Rev Biochem, 1990. 59: p. 439-73.

30. Sela-Culang, I., V. Kunik, and Y. Ofran, The structural basis of antibody-antigen recognition. Front Immunol, 2013. 4: p. 302.

31. Xu, J.L. and M.M. Davis, Diversity in the CDR3 region of $V(H)$ is sufficient for most antibody specificities. Immunity, 2000. 13(1): p. 37-45.

32. Monestier, M., et al., Structure and binding properties of monoclonal antibodies to core histones from autoimmune mice. Mol Immunol, 1993. 30(12): p. 1069-75.

33. Shi, P.T., et al., The antigenicity of myoglobin-related peptides synthesised on polyacrylamide and polystyrene resin supports. Mol Immunol, 1984. 21(6): p. 489-96.

34. Berglund, L., et al., A genecentric Human Protein Atlas for expression profiles based on antibodies. Mol Cell Proteomics, 2008. 7(10): p. 2019-27. 
1

2

3

4

5

6

7

8

9

10

11

12

13

14

15

16

17

18

19

20

21

22

23

24

25

26

27

28

29

30

31

32

33

34

35

36

37

38

39

40

41

42

43

44

45

46

47

48

49

50

51

52

53

54

55

56

57

58

59

60

35. Van Regenmortel, M.H., Requirements for empirical immunogenicity trials, rather than structure-based design, for developing an effective HIV vaccine. Arch Virol, 2012. 157(1): p. 1-20. 
Figure 1: Alignments of m-ras and h-ras primary amino acid sequences.

(A) m-ras (upper) complete amino acid sequence aligned to that of h-ras (lower). Spaces represent gaps in the alignment and conserved residues are highlighted by grey boxes (101 of 208 residues, $48.6 \%$ identity). Numbering is based upon m-ras primary sequence. (B) Alignment of C-terminal regions (186-208) of mras (upper) and corresponding region of h-ras (lower) with bolded residues representing sequences used for immunisation of rabbits and in peptide ELISA experiments (m-ras only). Grey boxes indicate that just 5 of 23 residues $(21.7 \%$ identity) are conserved.

Figure 2: ELISA to determine binding of rabbit antiserum to h-ras and m-ras peptides.

Diluted rabbit antiserum from an immunised rabbit was allowed to bind to plate-bound C-terminal m-ras peptide (KKKTKWRGDRATGTHKLQ) or the corresponding h-ras peptide (KLRKLNPPDESGPGCM) before bound rabbit IgG was detected.

\section{Figure 3: Amino acid and nucleotide alignments of rabbit anti-mras mAb variable regions.}

The rabbit anti-mras monoclonal antibody variable region sequences (mrasVk and mrasVh) are aligned with the closest rabbit germ-line segments as determined by IMGT/V-QUEST. (A) The anti-mras V region amino acid sequences are shown above the most likely germ-line sequences. Identical residues are shown with (-) and inserted or mutated residues highlighted in bold. Complementarity determining regions (CDR's) are highlighted grey and potential antigen-binding regions (ABRs) underlined. (B) The anti-mras $\mathrm{V}$ region CDR3 sequences are shown above the nucleotide sequence and most likely germ-line gene segment used during rearrangement. Mutated nucleotides and amino acid residues are shown in bold and junctional regions where $\mathrm{N}$-nucleotide addition could have resulted are highlighted.

Figure 4: ELISA to determine binding of rabbit anti-m-ras mAb to h-ras, r-ras2 and m-ras peptides.

Diluted culture supernatants containing rabbit mAb derived from an immunised rabbit was allowed to bind to plate-bound C-terminal m-ras peptide (KKKTKWRGDRATGTHKLQ), r-ras2 peptide (ECPPSPEPTRKEKDKKGCHC) or the corresponding h-ras peptide (KLRKLNPPDESGPGCM) before bound rabbit IgG was detected. Control refers to untransfected culture supernatant binding to plate-bound C-terminal m-ras peptide.

\section{Figure 5: ELISA to determine binding of rabbit anti-m-ras mAb to recombinant h-ras and m-ras.}

(A) Diluted purified rabbit $\mathrm{mAb}$ from an immunised rabbit was allowed to bind to plate-bound recombinant m-ras or to recombinant h-ras before bound rabbit IgG was assessed. (B) Recombinant h-ras and m-ras were captured with plate-bound scFv Y13-259 and diluted rabbit mAb (squares) or rabbit antisera (circles) added before bound rabbit IgG was assessed. 


\section{Figure 6: Peptide spot array of h-ras and m-ras.}

Binding of anti-rabbit IgG (secondary Ab only) and anti-mras mAb followed by secondary Ab (anti-mras $\mathrm{mAb}$ ) to h-ras peptides (upper) and m-ras peptides (lower). Numbering refers to peptide \# and sequence shown in Supplementary Figure 1. The primary sequence of h-ras is shorter so 91 peptides span this polypeptide whereas 99 peptides are required to span the entire m-ras polypeptide.

\section{Figure 7: Analysis of peptide spot array data.}

The h-ras (upper) and m-ras (lower) primary sequences are displayed with the overlapping peptides used in Figure 6 and shown in Supplementary Figure 1, represented by a line underneath. Binding of the anti-mras monoclonal IgG is represented as the relative spot intensity obtained (Fig 6). Each bar represents the level of IgG binding to the peptide and is aligned to the first residue of the peptide on the $\mathrm{x}$-axis i.e. for h-ras the first peptide with discernible binding was peptide 2 beginning with $\mathrm{E}$, whereas for $\mathrm{m}$-ras the first is peptide 4 beginning with $\mathrm{P}$.

\section{Supplementary Figure 1: Sequences of h-ras and m-ras peptide spots used in Figure 6.}

